\title{
Is Endoscopic Ultrasound-Guided Drainage Alone Sufficient for the Treatment of Peripancreatic Fluid Collection?
}

\author{
Se Woo Park \\ Division of Gastroenterology, Department of Internal Medicine, Hallym University Dongtan Sacred Heart Hospital, Hallym University College \\ of Medicine, Hwaseong, Korea
}

See "There Is No Advantage to Transpapillary Pancreatic Duct Stenting for the Transmural Endoscopic Drainage of Pancreatic Fluid Collections: A Meta-Analysis” by Sunil Amin, Dennis J. Yang, Aimee L. Lucas, et al., on page 388-394.

I read with great interest the paper by Amin et al., titled "There Is No Advantage to Transpapillary Pancreatic Duct Stenting for the Transmural Endoscopic Drainage of Pancreatic Fluid Collections: A Meta-Analysis" published in the July 2017 issue of Clinical Endoscopy. ${ }^{1}$ The authors concluded that transpapillary pancreatic duct (PD) drainage provides no additional clinical benefit for transmural (TM) peripancreatic fluid collections (PFC) and, therefore, patients with TM drainage may not require $\mathrm{PD}$ intervention.

However, I have a concern about the authors' conclusion, because the statistical significance was not identified in their meta-analyses between TM and CD. To interpret the results correctly, they had to say that "the clinical benefit of transpapillary pancreatic duct dranage in addition to the transmural dranage was not identified in our meta-analyses." In addition, confidence intervals were too wide in the meta-analyses. The 95\% confidence interval for technical success, for example, was $0.367-3.365$. Although the odds ratio was close to 1 , it was difficult to be sure that TM was equivalent to $\mathrm{CD}$ because of the wide confidence interval. More studies in this issue may

Received: June 22, 2017 Accepted: July 3, 2017

Correspondence: Se Woo Park

Division of Gastroenterology, Department of Internal Medicine, Hallym University Dongtan Sacred Heart Hospital, Hallym University College of Medicine, 7 Keunjaebong-gil, Hwaseong 18450, Korea

Tel: +82-31-8086-2858, Fax: +82-31-8086-2029, E-mail: britnepak@hallym.or.kr

(cc) This is an Open Access article distributed under the terms of the Creative Commons Attribution Non-Commercial License (http://creativecommons.org/ licenses/by-nc/3.0) which permits unrestricted non-commercial use, distribution, and reproduction in any medium, provided the original work is properly cited. change the conclusion.

Recently, endoscopic ultrasound (EUS)-guided drainage of the PFC has been widely accepted as a safe and effective standard technique compared with surgical approaches. Especially, EUS-guided drainage with a fully covered metal stent is associated with higher technical and functional success, shorter procedural time, and fewer short- and long-term adverse events. ${ }^{2}$ Among the several long-term adverse events, the major concern with this procedure is regarding the recurrence after the removal of TM stent. In one study reporting medium-term assessment of EUS-guided drainage of the PFC, ${ }^{3}$ the authors demonstrated that there were $6(10 \%)$ cases of symptomatic recurrence of PFC. In general, persistent or recurrent PFC might be attributed to PD injury or leakage. ${ }^{4}$ Therefore, any leakage observed during imaging before or after metal stent placement would require an additional procedure, such as PD stenting. According to some expert opinions, ${ }^{2}$ this strategy probably prevented further recurrence because the leakage healed by the time of follow-up, and the PD stent could be removed. This finding is similar to that of two recent studies ${ }^{5,6}$ in which an additional PD stent in patients with partial disruptions, but not in those with total disruptions, was found to improve the clinical outcomes compared with TM alone in patients with PFC. Furthermore, Trevino et al. reported that PD stenting might be one of the significant factors affecting the treatment success based on multi-variable analysis. ${ }^{5}$

From the opposite perspective, the technical difficulty of PD stenting in patients who underwent CD was theoretically higher compared to TM alone. Generally, the technical suc- 
cess rate of PD stenting varied from $17.5 \%$ to $40.2 \%,{ }^{5,7}$ because PD cannulation may be more difficult than biliary cannulation in some cases. Common causes of PD stenting failure include complete PD disruption, significant PD stricture or obstruction, inability to selectively perform deep PD cannulation, surgically altered anatomy, or luminal stricture that precludes the passage of the endoscope. ${ }^{6,8}$ Furthermore, difficult PD cannulation necessarily results in prolonged and frequent papillary manipulation, and repeated attempts at cannulation, regardless of the injection of contrast into the PD, are known to increase the risk of post-endoscopic retrograde cholangiopancreatography pancreatitis (PEP), especially in high-risk patients with one or more patient-related risk factors for PEP (such as younger age or female sex). ${ }^{9}$

In summary, there has been, to date, no evidence indicating which intervention is better regarding the efficacy and safety of PFC treatment through direct comparisons between TM alone and $\mathrm{CD}$. Thus, the choice of appropriate intervention for complete resolution and prevention of the recurrence of PFC should be made according to the status of PD disruption/leakage (partial or complete), technical difficulty of PD cannulation and stenting, characteristics and location of the PFC, and the possibility of recurrence. Additionally, further studies are needed to compare the efficacy and safety between direct methods, such as the transpapillary approach alone or EUS-guided TM approach alone and combined methods with transpapillary and TM approaches.

\section{Conflicts of Interest}

The author has no financial conflicts of interest.

\section{REFERENCES}

1. Amin S, Yang DJ, Lucas AL, Gonzalez S, DiMaio CJ. There is no advantage to transpapillary pancreatic duct stenting for the transmural endoscopic drainage of pancreatic fluid collections: a meta-analysis. Clin Endosc 2017;50:388-394.

2. Dhir V, Teoh AY, Bapat M, Bhandari S, Joshi N, Maydeo A. EUS-guided pseudocyst drainage: prospective evaluation of early removal of fully covered self-expandable metal stents with pancreatic ductal stenting in selected patients. Gastrointest Endosc 2015;82:650-657; quiz 718.e1-e5.

3. Ng PY, Rasmussen DN, Vilmann P, et al. Endoscopic ultrasound-guided drainage of pancreatic pseudocysts: medium-term assessment of outcomes and complications. Endosc Ultrasound 2013;2:199-203.

4. Arvanitakis M, Delhaye M, Bali MA, et al. Pancreatic-fluid collections: a randomized controlled trial regarding stent removal after endoscopic transmural drainage. Gastrointest Endosc 2007;65:609-619.

5. Trevino JM, Tamhane A, Varadarajulu S. Successful stenting in ductal disruption favorably impacts treatment outcomes in patients undergoing transmural drainage of peripancreatic fluid collections. J Gastroenterol Hepatol 2010;25:526-531.

6. Shrode CW, Macdonough P, Gaidhane M, et al. Multimodality endoscopic treatment of pancreatic duct disruption with stenting and pseudocyst drainage: how efficacious is it? Dig Liver Dis 2013;45:129133.

7. Varadarajulu S, Bang JY, Phadnis MA, Christein JD, Wilcox CM. Endoscopic transmural drainage of peripancreatic fluid collections: outcomes and predictors of treatment success in 211 consecutive patients. J Gastrointest Surg 2011;15:2080-2088.

8. Hookey LC, Debroux S, Delhaye M, Arvanitakis M, Le Moine O, Devière J. Endoscopic drainage of pancreatic-fluid collections in 116 patients: a comparison of etiologies, drainage techniques, and outcomes. Gastrointest Endosc 2006;63:635-643.

9. Testoni PA, Mariani A, Aabakken L, et al. Papillary cannulation and sphincterotomy techniques at ERCP: European society of gastrointestinal endoscopy (ESGE) clinical guideline. Endoscopy 2016;48:657-683. 